\title{
Lógica Difusa Basada en la Experiencia del Usuario para Medir la Usabilidad
}

\author{
Lionel Rodolfo Baquero Hernández, Osviel Rodríguez Valdés, Febe Ángel Ciudad Ricardo \\ Facultad 6 \\ Universidad de las Ciencias Informáticas \\ La Habana, Cuba \\ lrbaquero@estudiantes.uci.cu, \{osviel, fciudad\}@uci.cu
}

\begin{abstract}
Resumen - La mejor forma de crear un aplicación informática usable es realizando un diseño centrado en el usuario, diseñando para y por el usuario, en contraposición a lo que podría ser un diseño centrado en la tecnología o uno centrado en la creatividad u originalidad. Por lo que se puede también afirmar que la mejor forma de que la usabilidad sea medible es que dicha evaluación sea realizada por el usuario de la misma. Este trabajo se propone como objetivo diseñar una lógica difusa basada en la experiencia del usuario para medir la usabilidad. El trabajo con conjuntos difusos se acopla a la variabilidad de la medición de la usabilidad basada en la experiencia del usuario y reduce la necesidad de intervención de los expertos en la interpretación de los resultados de dicha medición. La teoría de la lógica difusa aplicada para realizar el análisis y evaluación de la usabilidad según la experiencia del usuario, genera y entrega datos más exactos, que otros métodos cualitativos. Esta brinda a los desarrolladores del software en cuestión la posibilidad de una mejor interpretación, libre de otras subjetividades.
\end{abstract}

Palabras clave- diseño centrado en el usuario, experiencia del usuario, lógica difusa, medir usabilidad, usabilidad.

\section{INTRODUCCIÓN}

La lógica (del griego logos: la razón, el principio que gobierna al universo): es un conjunto de reglas usadas para generar inferencias creíbles. El modelo aristotélico de razonamiento [1] se basa en el razonamiento exacto, es decir, una lógica dicotómica o binaria que admite dos posibilidades: verdadero-falso (o bien ceros y unos). Pero el mundo real es diferente, la información que de él obtenemos es incierta e imprecisa. Esto es válido para las ciencias naturales, humanísticas, ingeniería, derecho y medicina. La fuente de incertidumbre deriva, a grandes rasgos, de tres áreas: las deficiencias de la información (incompleta, errónea, imprecisa), las características propias del mundo real (no determinista) y las deficiencias de los modelos que intentan explicarlo (incompleto, inexacto).

En contraposición a la lógica dicotómica o binaria que admite dos posibilidades: verdadero- falso, la lógica multivaluada [2] admite varios valores de verdad posibles. La lógica difusa [3] (fuzzy logic) es una forma de lógica multivariada que intenta cuantificar esa incertidumbre. Ya no hay blancos y negros únicamente sino grises. La lógica difusa se utiliza cuando la complejidad del proceso en cuestión es muy alta y no existen modelos matemáticos precisos, para procesos altamente no lineales, y cuando se manejan definiciones y conocimiento no estrictamente definido (impreciso o subjetivo). Es importante señalar que, mientras la lógica difusa es un tema de gran actualidad, como lo prueba la intensa labor investigadora que se está realizando en ella, el método probabilista clásico y el modelo de factores de certeza se consideran temas muertos desde el punto de vista de la investigación [4].

Numerosos autores han propuesto diversas definiciones de usabilidad, normalmente a través de la enumeración de los diferentes atributos o factores mediante los que puede ser evaluada, dependiendo finalmente cada definición del enfoque con el que pretende ser medida [5]. La ISO 9241-11 [6] define la usabilidad como el grado de eficacia, eficiencia y satisfacción con la que usuarios específicos pueden lograr objetivos específicos, en contextos de uso específicos. También la ISO/IEC 9126 [7] plantea que la usabilidad es analizada en términos de su comprensibilidad, aprendizaje, operabilidad, atractivo y complacencia. La usabilidad se compone de dos tipos de atributos:

- Atributos cuantificables de forma objetiva: como son la eficacia o número de errores cometidos por el usuario durante la realización de una tarea, y eficiencia o tiempo empleado por el usuario para la consecución de una tarea.

- Atributos cuantificables de forma subjetiva: como es la satisfacción de uso, medible a través de la interrogación al usuario, y que tiene una estrecha relación con el concepto de Usabilidad Percibida [8].

La mejor forma de crear un aplicación informática usable es realizando un diseño centrado en el usuario, diseñando para y por el usuario, en contraposición a lo que podría ser un diseño centrado en la tecnología o uno centrado en la creatividad u originalidad [9]. Por lo que se puede también afirmar que la mejor forma de que la usabilidad sea medible es que dicha evaluación sea realizada por el usuario de la misma. Se puede deducir entonces que la usabilidad de un sistema informático está determinada por las condiciones específicas y concretas que circundan al usuario que la mide a partir de su experiencia, dado que esta puede variar en dependencia de quien la evalúe. Esperar siempre el mismo resultado independientemente de quien evalúe la usabilidad se corresponde con una visión generalizadora, lo cual es erróneo cuando se necesita una evaluación aterrizada al contexto específico del usuario. Se puede definir entonces usabilidad como una métrica de la calidad de un software que debe estar centrada en la experiencia del usuario y que puede variar en dependencia de quien la evalúe.

En este trabajo se propone como objetivo diseñar un sistema de lógica difusa basada en la experiencia del usuario para medir la usabilidad. Se espera que el trabajo con conjuntos difusos se acople al carácter variable de la medición de la usabilidad basada en la experiencia del usuario. Esta debe reducir la necesidad de intervención de los expertos en la interpretación de los resultados de dicha medición. 


\section{DEFINICIONES DE USABILIDAD}

Existen numerosas definiciones de usabilidad, esta investigación se sustentará sobre la base de las definiciones ofrecidas por la ISO 9241-11 e ISO/IEC 9126. De estas definiciones se tomarán los elementos que más se ajusten al objetivo de este trabajo y que puedan ser adaptados a la lógica difusa basada en la experiencia del usuario. Se pretende extraer de estas las métricas fundamentales para la evaluación de la usabilidad desde el punto de vista del usuario luego de su interacción con el software.

\section{A. Definición de ISO 9241-11}

El borrador internacional del estándar ISO/DIS 9241-11 (Guidance on Usability) [10] define cómo especificar y medir la usabilidad de productos y aquellos factores que tienen un efecto en la usabilidad. Para especificar o medir la usabilidad es necesario identificar las metas y descomponer la efectividad, eficiencia y satisfacción, así como los componentes del contexto de uso en subcomponentes con atributos medibles y verificables [11]:

- Eficacia: definido en términos de la exactitud y completitud con que usuarios específicos pueden lograr metas específicas en ambientes particulares.

- Eficiencia: referido a los recursos gastados en relación con la precisión y completitud de la meta lograda, es decir recursos de tiempo, financieros y humanos.

- Satisfacción: que evalúa el confort o comodidad y la aceptabilidad del trabajo del sistema para sus usuarios y otras personas afectadas por su uso.

En la Fig. 1 se muestra como la ISO 9241 define la usabilidad en términos de la calidad del trabajo de un sistema en uso y la interacción de sus componentes.

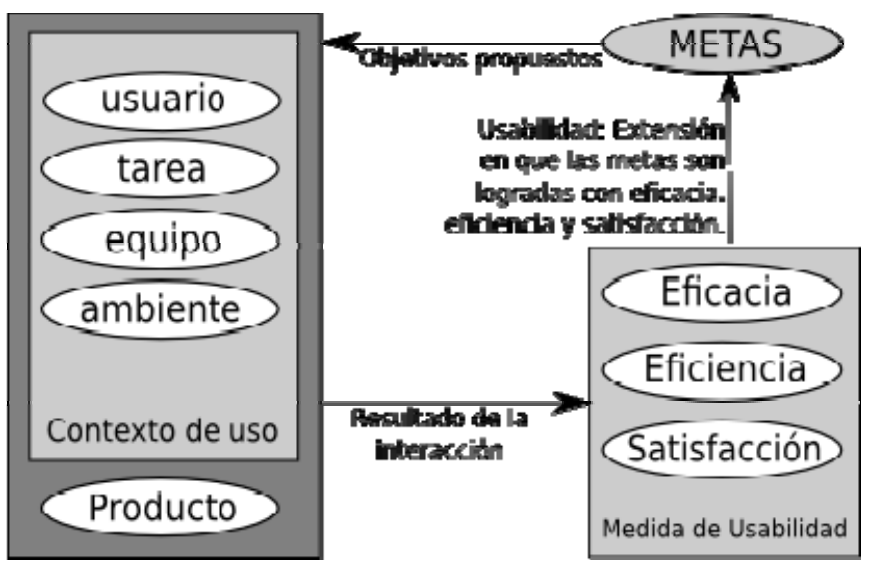

Figura 1. Marco de definición de usabilidad de ISO 9241-11.

Este marco permite visualizar todos los factores que pueden incidir sobre la usabilidad de un sistema en uso, desde una perspectiva real, lo cual es fundamental para determinar las necesidades reales de los usuarios. De esta definición se trabajará en la evaluación de la eficacia refiriéndose a la misma como la capacidad de la aplicación de permitirle al usuario completar las tareas que se propone. La eficiencia será evaluada en términos del esfuerzo y tiempo que demanda el software al usuario para realizar las tareas. La satisfacción del usuario para esta investigación representará, a criterio del usuario, la comodidad que este experimenta en el trabajo con el software.

\section{B. Definición de ISO/IEC 9126}

De acuerdo al estándar ISO/IEC 9126 [7], usabilidad es un atributo de la calidad del software, definida un conjunto de atributos de software que se sostienen en el esfuerzo necesitado para el uso y en la valoración individual de tal uso por un conjunto de usuarios declarados o implicados. En este estándar se incluyen las siguientes métricas [12]:

- Comprensibilidad, define la capacidad del producto software para permitir al usuario entender si el software es adecuado, y cómo puede ser usado para tareas y condiciones de uso particulares.

- Aprendizaje, referido a la capacidad del producto software para permitir a los usuarios aprender a usar sus aplicaciones.

- Operabilidad, es la capacidad del producto software para permitir al usuario operarlo y controlarlo. Aspectos de conformidad, mutabilidad, adaptabilidad e instalación pueden afectar a la operabilidad. También este atributo corresponde a la tolerancia de error, y conformidad con las expectativas del usuario. En un sistema, sobre el que opera un usuario, la combinación de funcionalidad, confiabilidad, usabilidad y eficiencia pueden ser medidas externamente por la calidad de uso.

- Atractivo, es la capacidad del producto software para ser atractivo al usuario. Está referido a los atributos del software pensados para hacer el software más atractivo al usuario, tal como el uso de color y la naturaleza del diseño gráfico.

- Conformidad a estándares y pautas, referido a la capacidad del producto software para adherirse a estándares, convenciones, guías de estilo o regulaciones relacionadas con la usabilidad.

Se utilizarán estas métricas para la evaluación de la usabilidad basada en la experiencia del usuario que interactúa con un software. Estas son una base fundamental para determinar la consideración del usuario acerca de la usabilidad del producto software en cuestión. Ayudarán a determinar la capacidad del software para ser entendido, aprendido, usado y atractivo para el usuario, cuando es utilizado bajo las condiciones específicas del mismo. También se deberá tener en cuenta la conformidad del software a los estándares, políticas y regulaciones del contexto en el que se desarrolla el negocio del cual es parte el usuario. En la Fig. 2 se muestra como la usabilidad es incluida por la ISO/IEC 9126 como un factor de calidad.

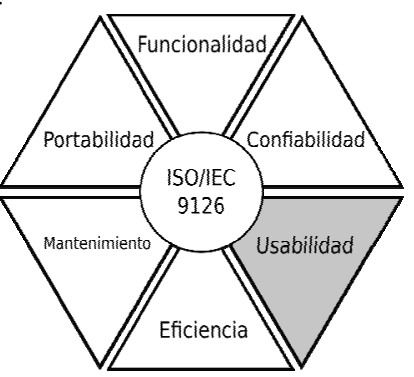

Figura 2. La usabilidad como factor de calidad de software de acuerdo a la ISO/IEC 9126

\section{EVALUACIÓN DE LA USABILIDAD}

La evaluación de la usabilidad es un proceso para producir una medida de la facilidad de uso. En la evaluación, hay un objeto que está siendo evaluado y un proceso a través del cual uno o más atributos son juzgados o se les da un valor [13]. La evaluación de usabilidad para algunos autores como Mayhew [14], es un estudio empírico con usuarios reales del sistema propuesto, con el propósito de proporcionar retroalimentación en el desarrollo de software durante el ciclo de vida de desarrollo iterativo. La evaluación de la usabilidad, es una 
tarea de gran importancia cuando se está desarrollando un software. Para la presente investigación se realizará la evaluación de la usabilidad mediante Cuestionario [15]. A los usuarios que interactúan con el software se deberá realizar la encuesta de la Tabla I. Al asignarle un valor alto a uno de los indicadores, el usuario estará manifestando una alta satisfacción en ese aspecto. Por el contrario una baja evaluación representa una inconformidad latente en ese indicador.

Al aplicar la encuesta al usuario se le debe explicar claramente que debe, para cada uno de los indicadores asignarle una evaluación entre cero (0) y diez (10), según su experiencia del trabajo con el software. Se le debe explicar además, que los valores más altos en esta escala representan mayor satisfacción. Esta encuesta se basa solo en su criterio como usuario del software que se está desarrollando, por lo que su opinión no debe estar determinada por ningún factor ajeno a su propio criterio.

TABLA I. ENCUESTA PARA MEDIR LA USABILIDAD.

\begin{tabular}{lll}
\hline \hline No. & \multicolumn{1}{c}{ Indicador } & Evaluación \\
\hline \hline 1 & $\begin{array}{l}\text { El software me permite completar las tareas que deseo } \\
\text { realizar. }\end{array}$ \\
2 & El software me permite ahorrar tiempo y esfuerzo en la \\
& realización de las tareas. \\
3 & Mi satisfacción con la comodidad en el trabajo con el \\
& software. \\
4 & El software es comprensible para mi. \\
5 & El software me permite aprender a usarlo. \\
6 & El software me permite operarlo y controlarlo. \\
7 & El software me resulta atractivo. \\
8 & El software se ajusta a las políticas, estándares y pautas \\
& de los procesos que automatiza.
\end{tabular}

La encuesta se compone de 8 preguntas orientadas a que el usuario luego de interactuar con el software en cuestión pueda dar su criterio sobre cada una de las métricas o indicadores referentes a la usabilidad, según las definiciones de la ISO 9241-11 y la ISO/IEC 9126. Los indicadores número 1, 2 y 3 están asociados a las métricas de eficacia, eficiencia y satisfacción definidas por la ISO 6241-11 respectivamente. Los indicadores número 3, 4, 5, 6 y 7 están relacionados con las métricas de comprensibilidad, aprendizaje, operabilidad, atractivo y conformidad, en ese mismo orden.

\section{LÓGICA DIFUSA}

La lógica difusa es un modo de razonamiento que aplica valores múltiples de verdad o confianza a las categorías restrictivas durante la resolución de problemas. En ella se descubren grados diversos de pertenencia y no adscripciones basadas en todo o nada [16]. En los conjuntos difusos un elemento puede pertenecer simultáneamente a dos conjuntos, dependiendo de su grado de pertenencia, el cual se encontrará en el intervalo $[0,1]$. Mientras más cercano esté el valor a 0 menos podemos asegurar la pertenencia de un elemento a un conjunto. Por el contrario cuanto más cercano esté el valor a 1 más podemos asegurar la pertenencia del elemento al conjunto.

Las variables lingüísticas son aquellas variables de lenguaje natural caracterizadas por los conjuntos difusos definidos en el universo del discurso en la cual se encuentra definidas [17]. Son conceptos que no se clasifican con valores de verdad absoluto, se clasifican mediante conjuntos difusos. Son sustantivos que reflejan una propiedad de un elemento. A estas variables se les pueden asignar etiquetas, que no son más que las posibles clasificaciones de la variable. Se refieren a los posibles adjetivos con los que puedan ser clasificadas las variables lingüísticas. El intervalo en el que oscilarán los posibles valores de las variables lingüísticas será llamado universo discurso.

\section{A. Funciones típicas de pertenencia.}

El grado de pertenencia de un elemento $\mathrm{M}(\mathrm{x})$ a un conjunto difuso será determinado por funciones de pertenencia. Las funciones típicas de pertenencia más usadas según [18] son Función L, Función GAMMA, Función LAMBDA y Función PI. Las funciones de pertenencia de cada una de estas funciones se pueden observar en la Tabla II, que se muestra.

\section{TABLA II. CÁLCULO DE GRADO DE PERTENENCIA CON} FUNCIONES TÍPICAS.

\begin{tabular}{|c|c|c|c|}
\hline \multicolumn{2}{|c|}{ Funciones típicas } & \multicolumn{2}{|c|}{ Grado de pertenencia } \\
\hline \multirow{3}{*}{$\begin{array}{r}M(x) \\
1\end{array}$} & FUNCIÓN L & $\mathrm{M}(\mathrm{x})=1$ & si $\mathrm{X} \leq \mathrm{A}$ \\
\hline & & $\mathrm{M}(\mathrm{x})=(\mathrm{B}-\mathrm{X}) /(\mathrm{B}-\mathrm{A})$ & si $\mathrm{A}<\mathrm{X}<\mathrm{B}$ \\
\hline & & $M(x)=0$ & si $X \geq B$ \\
\hline & B & & \\
\hline \multirow[t]{3}{*}{$M(x) \uparrow$} & FUNCIÓN GAMMA & $\mathrm{M}(\mathrm{x})=0$ & si $\mathrm{X} \leq \mathrm{A}$ \\
\hline & & $M(x)=(X-A) /(B-A)$ & si $\mathrm{A}<\mathrm{X}<\mathrm{B}$ \\
\hline & & $M(x)=1$ & si $X \geq B$ \\
\hline & B & & \\
\hline
\end{tabular}

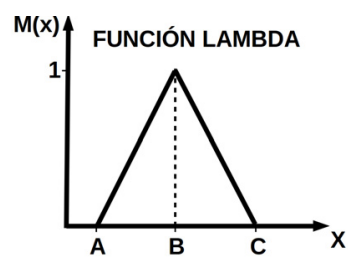

$$
\begin{array}{ll}
M(x)=0 & \text { si } X \leq A \text { o } X \geq C \\
M(x)=(X-A) /(B-A) & \text { si } X>A \text { y } X \leq B \\
M(x)=(C-X) /(C-B) & \text { si } X>B \text { y } X<C
\end{array}
$$

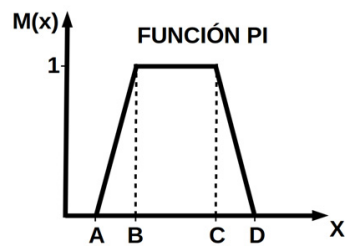

$$
\begin{array}{ll}
\mathrm{M}(\mathrm{x})=0 & \text { si } \mathrm{X} \leq \mathrm{A} \text { o } \mathrm{X} \geq \mathrm{C} \\
\mathrm{M}(\mathrm{x})=(\mathrm{X}-\mathrm{A}) /(\mathrm{B}-\mathrm{A}) & \text { si } \mathrm{A}<\mathrm{X}<\mathrm{B} \\
\mathrm{M}(\mathrm{x})=1 & \text { si } \mathrm{B} \leq \mathrm{X} \leq \mathrm{C} \\
\mathrm{M}(\mathrm{x})=(\mathrm{D}-\mathrm{X}) /(\mathrm{D}-\mathrm{C}) & \text { si } \mathrm{C}<\mathrm{X}<\mathrm{D}
\end{array}
$$

De estas 4 funciones típicas de pertenencia que se han descrito se utilizarán en la lógica difusa para la evaluación de la usabilidad las correspondientes con las funciones L, GAMMA y PI. Serán utilizadas para el proceso de Fuzzyficación de los indicadores de medición de la usabilidad del software que fueron definidos para la encuesta realizada al usuario. 


\section{B. Operaciones sobre sonjuntos difusos.}

Sobre los conjuntos difusos se pueden realizar operaciones lógicas de intersección (conjunción), la unión (disyunción) y el complemento (negación). Para hacer dichas operaciones se pueden utilizar las T-Normas y las S-Normas. Las T-Normas especifican las condiciones que deben reunir las operaciones para interceptar conjuntos y las S-Normas lo hacen para las uniones. Las intersecciones ocurren en las conjunciones (CONJ) y las contribuciones (CTR), de forma parecida las uniones ocurren en las disyunciones (DISY) y el global (GLOB). Estas operaciones son realizadas en los sistemas expertos [19] para calcular los factores de certeza de las reglas de producción. Según las T-Normas y las S-Normas estas operaciones se pueden realizar según se describe en la Tabla III.

TABLA III. FUNCIONES PARA EL CÁLCULO DE T-NORMAS Y SNORMAS.

\begin{tabular}{ccc}
\hline \hline T-Norma(x,y) & S-Normas(x.y) & Nombre del par T-S \\
\hline \hline $\min (\mathrm{x}, \mathrm{y})$ & $\max (\mathrm{x}, \mathrm{y})$ & Mínimo-Máximo \\
$\mathrm{x} * \mathrm{y}$ & $\mathrm{x}+\mathrm{y}-\mathrm{x} * \mathrm{y}$ & Probabilístico \\
$\max (0, \mathrm{x}+\mathrm{y}-1)$ & $\min (1, \mathrm{x}+\mathrm{y})$ & Lukasiewicz \\
\hline
\end{tabular}

En un modelo difuso T-Normas se utilizan para calcular CONJ y CTR, mientras que las S-Normas para las DISY y GLOB. Para el cálculo del complemento (NEG) se usará la función $\operatorname{NEG}(\mathrm{x})=1-\mathrm{x}$.

En el cálculo de factor de certeza la operación CTR se utilizará conociendo el factor de certeza de la regla de producción a la que pertenece y los factores de certezas de las premisas de dicha regla. A partir de esto se podrá inferir el factor de certeza de las conclusiones de la regla. Por su parte la operación GLOB se utilizará para determinar que factor de certeza asumir para una conclusión que es inferida de más de una regla de producción. Es importante acotar que en los casos en que no se tiene el factor de certeza para una regla se asume el máximo valor que este puede tomar, que es 1 .

\section{Esquema general de un sistema de lógica difusa.}

En un sistema de lógica difusa se tienen variables lingüísticas, sus etiquetas, las funciones de pertenencia de las etiquetas, las reglas de producción y los factores de certeza asociados a estas reglas. Como datos de entrada al sistema se tienen valores numéricos que toman las variables lingüísticas. Estos valores se convierten en valores de pertenencia a etiquetas difusas que son equivalentes a los factores de certeza. Este proceso se llama Fuzzyficación, dado que convierte valores numéricos a difusos. A partir de estos valores obtenidos en el proceso de Fuzzyficación ocurre el proceso de propagación de certeza usando las reglas de producción definidas. Este es el proceso de Inferencia Fuzzy, en el cual se utilizan las funciones de las T-Normas y las SNormas. Obteniéndose como resultados valores de certeza que se refieren a las pertenencias a los conjuntos de salida. Por último a partir de los valores de pertenencia a las variables lingüísticas de salida hay que obtener los valores numéricos de estas y a este proceso se le denomina Desfuzzyficación. La Desfuzzyficación de las variables puede realizarse, entre otros procedimientos por el Método del Centroide (COG), el más utilizado para este proceso. Para entender mejor lo anteriormente descrito se puede observar la Fig. 3.

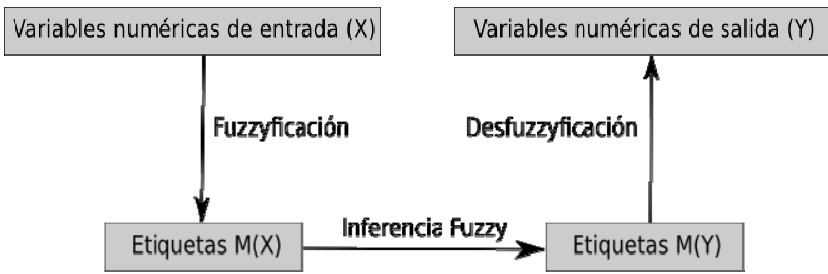

Figura 3. Esquema de un sistema difuso.

\section{Método del Centroide.}

El Método del Centroide o Centro de Gravedad (COG) será usado para Desfuzzyficar numéricamente el valor de la Usabilidad del software, de forma similar a la forma en que fue utilizado por [20]. Lo interesante de este método es que no hay que ajustar ningún coeficiente, solo es necesario conocer las funciones de pertenencia de cada una de las etiquetas definidas. El método COG igualmente parte de los valores de pertenencia a cada una de las etiquetas asociadas a la variable que se quiere Desfuzzyficar.

Para cada variable de salida fuzzyficada, se trunca el valor máximo de la función de pertenencia de cada etiqueta, a partir del valor obtenido durante la inferencia. El efecto de este truncamiento se observa en gráfico de la Fig. 4. Lo mismo se hace para cada etiqueta. Cada una se trunca según el valor de certeza inferido. De esta manera, son más truncados los gráficos de las etiquetas inferidos con menor valor.

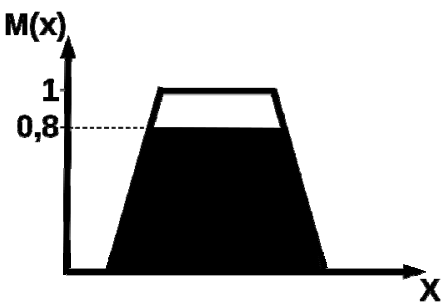

Figura 4. Truncamiento del conjunto difuso según el factor de certeza de la variable de salida para este.

Luego se combina el resultado del truncamiento de todas estas funciones y se obtiene el centro de gravedad. Para eso se usa la expresión:

$$
C O G=\frac{\int M(x) * x d x}{\int M(x) d x}
$$

Donde $\mathrm{M}(\mathrm{x})$, como ya se ha mencionado, representará el grado de pertenencia del elemento $\mathrm{X}$ que tomará valores en el universo discurso, usando un paso definido por el usuario. Mientras menor sea este paso más exacto será el resultado del COG.

\section{LÓGICA DIFUSA PARA MEDIR LA USABILIDAD}

Para medir la usabilidad usando lógica difusa se tendrán como variables lingüísticas los indicadores correspondientes a la encuesta realizada al usuario (Ver Tabla I). Estos indicadores son la eficacia, eficiencia, satisfacción, comprensibilidad, aprendizaje, operabilidad, atractivo y conformidad. Como variable de salida se tiene la Usabilidad. Se definió que cada una de estas variables de entrada o salida, tendrán asociadas las etiquetas de BAJA, MEDIA, ALTA y EXCELENTE. Para valorar el impacto que tienen las etiquetas lingüísticas de la variable de salida se debe consultar la Tabla IV. 
TABLA IV. IMPACTO DE LAS ETIQUETAS DE LA VARIABLE DE SALIDA.

\begin{tabular}{cl}
\hline \hline ETIQUETA & \multicolumn{1}{c}{ IMPACTO } \\
\hline \hline BAJA & $\begin{array}{l}\text { Se requiere de acciones para cambiar el diseño e } \\
\text { implementación del software. }\end{array}$ \\
MEDIA & $\begin{array}{l}\text { Se requiere de accionnes para mejorar el diseño e } \\
\text { implementación del software. }\end{array}$ \\
ALTA & $\begin{array}{l}\text { Se puede valorar la implementación de accionnes para } \\
\text { mejorar aspectos mínimos del diseño e implementación } \\
\text { del software. }\end{array}$ \\
EXCELENTE & $\begin{array}{l}\text { No se requiere de acciones para cambiar el diseño e } \\
\text { implementación del software. }\end{array}$ \\
\hline
\end{tabular}

Para la etiqueta BAJA la función de pertenencia asociada será la función $\mathrm{L}$, tal que $<0,0,4,5>$. El primer valor representa dónde comienza la función, el segundo dónde se hace 1, el tercero dónde comienza a disminuir y el cuarto dónde se hace 0 . Para la etiqueta MEDIA, utilizando la función PI, se tiene $<4,5,6,7>$. Para la etiqueta ALTA, de función de distribución PI igualmente, será $\langle 6,7,8,9\rangle$. Y para la etiqueta EXCELENTE, mediante la función Gamma, se podrá representar a través de $<8,9,10,10>$. La Fig. 5 muestra las funciones de pertenencia de las etiquetas lingüísticas de las variables de entrada.

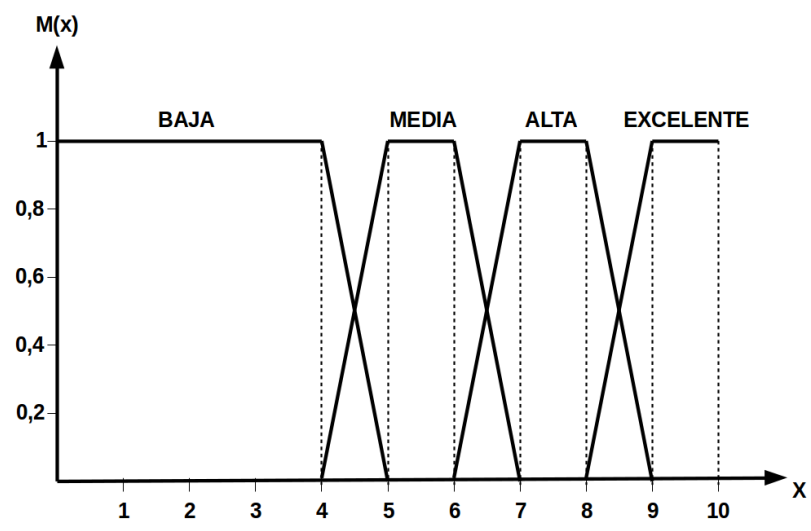

Figura 5. Funciones de pertenencia de las etiquetas lingüísticas de las variables de entrada.

Utilizando le valoración de expertos en el tema se definieron las reglas de producción. Estas reglas garantizan que siempre la evaluación de la Usabilidad esté mayormente determinada por la menor evaluación obtenida en los indicadores de entrada. Las reglas de producción y los factores de certeza asociados a cada una fueron definidos de la siguiente forma:

- R1: Si la eficacia es baja (EB) o eficiencia es baja (EFB) o la satisfacción es baja (SB) o la comprensibilidad es baja (CMB) o el aprendizaje es bajo (APB) o la operabilidad es baja (OB) o el atractivo es bajo (ATB) o la conformidad es baja (CNB), entonces la Usabilidad es baja (UB). ( $\mathrm{FC}=1)$

- R2: Si la eficacia es media (EM) o eficiencia es media (EFM) o la satisfacción es media (SM) o la comprensibilidad es media (CMM) o el aprendizaje es medio (APM) o la operabilidad es media (OM) o el atractivo es medio (ATM) o la conformidad es media (CNM), entonces la Usabilidad es media (UM). $(\mathrm{FC}=0,8)$

- R3: Si la eficacia es alta (EA) o eficiencia es alta (EFA) o la satisfacción es alta (SA) o la comprensibilidad es alta (CMA) o el aprendizaje es alto (APA) o la operabilidad es alta (OA) o el atractivo es alto (ATA) o la conformidad es alta (CNA), entonces la Usabilidad es alta (UA). $(\mathrm{FC}=0,6)$

- R4: $\mathrm{Si}$ la eficacia es excelente (EE) o eficiencia es excelente (EFE) o la satisfacción es excelente (SE) o la comprensibilidad es excelente (CME) o el aprendizaje es excelente (APE) o la operabilidad es excelente (OE) o el atractivo es excelente (ATE) o la conformidad es excelente (CNE), entonces la Usabilidad es excelente (UE). $(\mathrm{FC}=0,4)$

Una vez que se tienen estos datos se podrá proceder a la Fuzzyficación de las variables de entrada. El universo discurso es igual para todas las variables de entrada que se han definido, por lo que todas las variables de entrada tienen las mismas etiquetas lingüísticas y funciones de pertenencia.

Luego de calcular los factores de certeza para cada una de las etiquetas de las variables de entrada, se pasará a la fase de Inferencia Fuzzy. En esta se calcularán los factores asociados a las etiquetas de las variables de salida. A partir de las cuatro reglas de producción definidas se calcularán las DISY y CTR necesarias, siguiendo el par Mínimo-Máximo de las T-Normas y S-Normas.

En la tercera fase se procederá a la Desfuzzyficación que se realizará mediante el Método del Centroide. El paso será de 1 , dado que $\mathrm{x}$ irá desde $\mathrm{X}_{1}$ hasta $\mathrm{X}_{10}$, para ganar en exactitud en la medida de la Usabilidad. Las etiquetas de la variable de salida Usabilidad serán las mismas utilizadas para las variables de entrada, al igual que sus funciones de pertenencia. En la Fig. 6 se muestra el esquema general de la lógica difusa basada en la experiencia del usuario para medir la usabilidad.

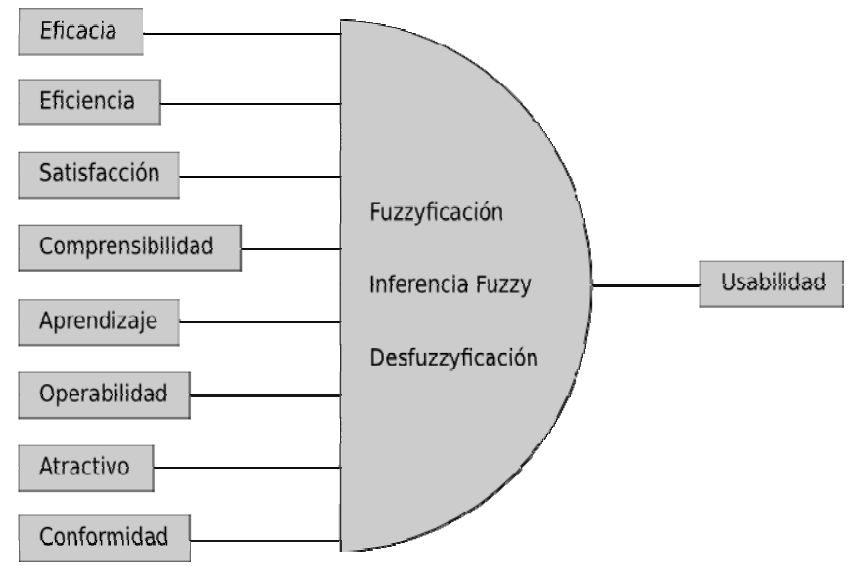

Figura 6. Esquema general de la lógica difusa basada en la experiencia del usuario para medir la usabilidad.

\section{RESULTADOS Y DISCUSIÓN}

Para evaluar los resultados de la presente investigación se realizará una experimentación [21]. El experimento que se desarrollará tendrá como principal objetivo demostrar la efectividad de la lógica difusa basada en la experiencia del usuario para medir la usabilidad. Para ello se simulará el proceso completo para un usuario. Una vez concluido este proceso se hará la discusión de los resultados de la experimentación.

Se tienen los valores de entrada $[2,2,5,4,3,4,5,3]$ para los indicadores de eficacia, eficiencia, satisfacción, comprensibilidad, aprendizaje, operabilidad, atractivo y conformidad respectivamente. En el proceso de Fuzzyficación se calculan los FC de cada una de las variables de entrada para cada una de sus etiquetas. Al aplicarle la Fuzzyficación a las variables de entrada, teniendo los valores numéricos los asociados a cada una de ellas, se obtienen los resultados de la 
Tabla V. El cálculo de los grados de pertenencia se realiza según las funciones típicas de pertenencia.

TABLA V. GRADOS DE PERTENENCIA DE LOS VALORES DE ENTRADA A LOS CONJUNTOS DIFUSOS.

\begin{tabular}{ccccc}
\hline $\begin{array}{c}\text { Variable } \\
\text { lingǘstica }\end{array}$ & $\begin{array}{c}\text { Etiqueta } \\
\text { BAJA }\end{array}$ & $\begin{array}{c}\text { Etiqueta } \\
\text { MEDIA }\end{array}$ & $\begin{array}{c}\text { Etiqueta } \\
\text { ALTA }\end{array}$ & $\begin{array}{c}\text { Etiqueta } \\
\text { EXCELENTE }\end{array}$ \\
\hline Eficacia & 1 & 0 & 0 & 0 \\
Eficiencia & 1 & 0 & 0 & 0 \\
Satisfacción & 0 & 1 & 0 & 0 \\
Comprensibilidad & 1 & 0 & 0 & 0 \\
Aprendizaje & 1 & 0 & 0 & 0 \\
Operabilidad & 1 & 0 & 0 & 0 \\
Atractivo & 0 & 1 & 0 & 0 \\
Conformidad & 1 & 0 & 0 & 0 \\
\hline
\end{tabular}

El proceso de Inferencia Fuzzy se realiza a través de las reglas definidas, utilizando el par Mínimo-Máximo de las TNormas y S-Normas. Una vez realizado este proceso se obtienen los valores que se muestran en la Tabla VI para la variable de salida Usabilidad.

TABLA VI. GRADOS DE PERTENENCIA DE LA VARIABLE DE SALIDA A LOS CONJUNTOS DIFUSOS.

\begin{tabular}{ccccc}
\hline $\begin{array}{c}\text { Variable } \\
\text { lingüística }\end{array}$ & $\begin{array}{c}\text { Etiqueta } \\
\text { BAJA }\end{array}$ & $\begin{array}{c}\text { Etiqueta } \\
\text { MEDIA }\end{array}$ & $\begin{array}{c}\text { Etiqueta } \\
\text { ALTA }\end{array}$ & $\begin{array}{c}\text { Etiqueta } \\
\text { EXCELENTE }\end{array}$ \\
\hline Usabilidad & 1 & 0,8 & 0 & 0 \\
\hline
\end{tabular}

La Inferencia Fuzzy arroja como resultados que el grado de pertenencia de la variable de salida es de 1 para la etiqueta BAJA y de 0,8 para la etiqueta MEDIA, por lo tanto para los valores de entrada la Usabilidad será BAJA. Para Desfuzzyficar la variable de salida se aplica el Método del Centroide, con el cual se obtiene el valor que se muestra en la Tabla VII.

TABLA VII. RESULTADOS DE LA APLICACIÓN DEL MÉTODO DEL CENTROIDE.

\begin{tabular}{rcc}
\hline \hline $\mathbf{x}_{\mathbf{i}}$ & $\mathbf{M}\left(\mathbf{x}_{\mathbf{i}}\right)$ & $\mathbf{x}_{\mathbf{i}}{ }^{*} \mathbf{M}\left(\mathbf{x}_{\mathbf{i}}\right)$ \\
\hline 1 & 1 & 1 \\
2 & 1 & 2 \\
3 & 1 & 3 \\
4 & 1 & 4 \\
5 & 0,8 & 4 \\
6 & 0,8 & 4,8 \\
7 & 0 & 0 \\
8 & 0 & 0 \\
9 & 0 & 0 \\
10 & 0 & 0 \\
\hline SUMA & 5,6 & 18,8 \\
\hline COG & & $\mathbf{3 , 3 6}$ \\
\hline
\end{tabular}

En la Fig. 7 se muestra el grado de pertenencia de la variable de salida (Usabilidad) con valor 3,36 para los valores de entrada $([2,2,5,4,3,4,5,3])$ correspondientes. Aquí se observa que para el valor de esta variable, el grado de pertenencia de a la etiqueta lingüística BAJA es del $100 \%$, lo cual significa que se requiere de acciones para cambiar el diseño e implementación del software, según lo establecido en la Tabla IV.

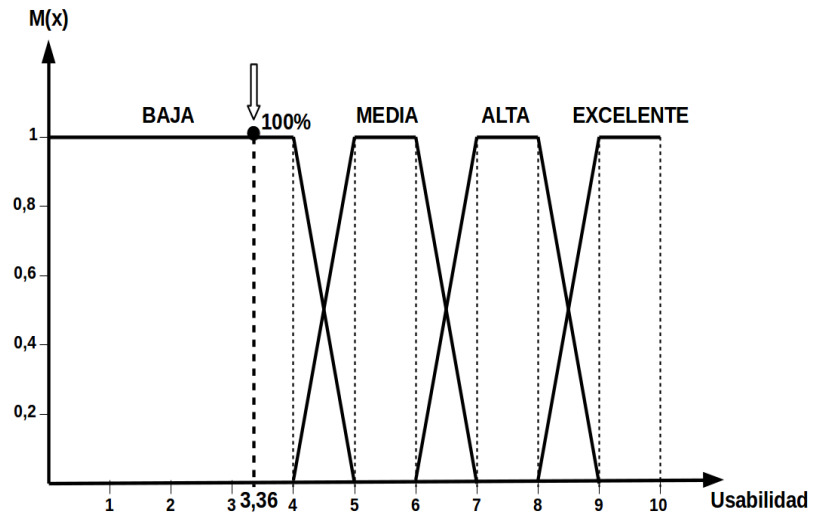

Figura 7. Grado de pertenencia de la variable Usabilidad para los valores de entrada $[2,2,5,4,3,4,5,3]$.

\section{CONCLUSIONES}

La teoría de la lógica difusa aplicada para realizar el análisis y evaluación de la usabilidad según la experiencia del usuario, genera y entrega datos más exactos, que otros métodos cualitativos. Esta brinda a los desarrolladores del software en cuestión la posibilidad de una mejor interpretación, libre de otras subjetividades. Una vez analizados los resultados de la investigación se puede afirmar que se ha dado cumplimiento al objetivo de la misma, lográndose el diseño de un sistema de lógica difusa capaz de medir la usabilidad a partir de la experiencia del usuario. La lógica difusa se posiciona como una alternativa para el desarrollo de sistemas y herramientas, que sirven de apoyo a la toma de decisiones empresariales en lo referente a la calidad de software.

\section{REFERENCIAS}

[1] Martínez, O., \& María, J. El papel del razonamiento analógico en la construcción histórica de la noción de fuerza gravitatoria y del modelo del sistema solar (Primera Parte). Revista Eureka sobre Enseñanza y divulgación de las Ciencias, 2004, 1.

[2] Serres, F. M. Proof Procedures for Multiple-Valued Propositional Logics (Doctoral dissertation, Universitat Autònoma de Barcelona), 1997.

[3] Morales Luna, G. Introducción a la lógica difusa. Centro de Investigación y Estudios Avanzados. México, 2002.

[4] DNegri, C. E., \& De Vito, E. L. Introducción al razonamiento aproximado: lógica difusa. Revista Argentina de medicina respiratoria, 2006, 4, 126-136.

[5] Folmer, E., Bosch, J. Architecting for usability: a survey. En: Journal of Systems and Software. Febrero 2004, v. 70, n. 1-2. pp. 61-78.

[6] ISO 9241-11. Ergonomic requirements for office work with visual display terminals (VDT)s - Part 11 Guidance on usability, 1998.

[7] Jung, H. W., Kim, S. G., \& Chung, C. S. Measuring software product quality: A survey of ISO/IEC 9126. IEEE software, 2004, (5), 88-92. 
[8] Hassan, Y., Martín Fernández, F. J., \& Iazza, G. Diseño web centrado en el usuario: usabilidad y arquitectura de la información. Hipertext. Net, 2004, (2).

[9] Hassan Montero, Y. Introducción a la Usabilidad. No sólo usabilidad, 2002, (1).

[10] ISO CD 9241-11. Guidelines for specifying and measuring usability, 1993.

[11] Obeso, M. E. A. Metodología de medición y evaluación de la usabilidad en sitios web educativos, 2005, (p. 31). Universidad de Oviedo.

[12] Bevan, N., \& Azuma, M. Quality in use: Incorporating human factors into the software engineering lifecycle. In Software Engineering Standards Symposium and Forum. Emerging International Standards. ISESS 97., Third IEEE International, 1997, (pp. 169-179). IEEE.

[13] Karat, J. User-centered software evaluation methodologies. In M. Helander, T.K. Landauer, P. Prabhu (Eds.) Handbook of human-computer interaction, 1997, 2nd ed. Elsevier Science.

[14] Mayhew, D. The Usability Engineering Lifecycle. Morgan Kaufmann, San Francisco, California, 1999.

[15] Granollers, T., \& Lorés, J. Esfuerzo de Usabilidad: un nuevo concepto para medir la usabilidad de un sistema interactivo basada en el Diseño Centrado en el Usuario. En V Congreso Interacción Persona Ordenador, 2004.

[16] Brage, L. B. and Cañellas, A. J. C. Lógica difusa: una nueva epistemología para las Ciencias de la Educación. Revista de educación, 2006, (340), 995-1008.

[17] Salas, A. G. Lógica Difusa. Revista de Información, Tecnología y Sociedad, 2008, 122.

[18] Ramón, Y. E. S. Diseño de un controlador de temperatura difuso. Revista Matices tecnológicos, 2010, 2.

[19] Sierra, E. A. et al. Sistemas expertos que recomiendan estrategias de instrucción. Un modelo para su desarrollo. Revista Latinoamericana de Tecnología Educativa-RELATEC, 2007, 1(1), 33-47.

[20] Angarita, A. A. et al. Definición de un modelo de medición de análisis de riesgos de la seguridad de la información aplicando lógica difusa y sistemas basados en el conocimiento1 Definition of a model for measuring risk analysis. Comité Editorial, 2015, 71.

[21] Sampieri, R., Collado, C., \& Lucio, P. Metodología de la investigación. Mc Graw Hill. 2006. 850p.

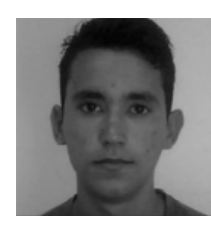

Lionel Rodolfo Baquero Hernández, estudiante de 5 to año de Ingeniería en Ciencias Informáticas, Facultad 6, Universidad de las Ciencias Informáticas. La Habana, Cuba. Sus áreas de investigación son la Ingeniería de Software, los Procesos de Negocio y la Inteligencia Artificial.

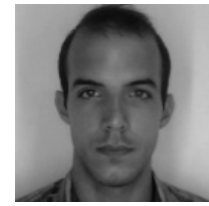

Osviel RodriguezValdés, Ingeniero en Ciencias Informáticas por Universidad de las Ciencias Informáticas. Profesor del departamento de Programación y Señales Digitales. Sus áreas de investigación son la Ingeniería de Software, los Procesos de Negocio y la Inteligencia Artificial.

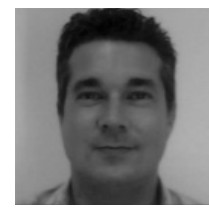

Febe Ángel Ciudad Ricardo, graduado como Ingeniero Informático en el año 2004 por la Universidad de Holguín. Titulado como Máster en Informática Aplicada en el año 2007 por la Universidad de las Ciencias Informáticas (UCI) y obtuvo el grado científico de Doctor en Ciencias de la Educación en el año 2012 por la Universidad de La Habana (UH). Imparte docencia de pregrado como profesor asistente en las disciplinas de Ingeniería y Gestión de Software, Metodología de la Investigación Científica y Formación Pedagógica. Es miembro de los claustros de las maestrías de Informática Aplicada, Informática Avanzada, Gestión de Proyectos y Educación a Distancia de la UCI. Desarrolla sus investigaciones en las temáticas de Ingeniería y Gestión de Software, con énfasis en el área del Software Educativo; así como en la Tecnología e Informática Educativas. Ha publicado diversos artículos científicos y ha participado en diferentes eventos nacionales e internacionales en estas áreas del conocimiento. Ha sido arquitecto, analista y líder de proyectos de desarrollo de software, jefe de departamento docente y asesor técnico - docente. Se desempeñó como Director del Centro de Innovación y Calidad de la Educación (CICE) de la UCI por varios años. Actualmente se desempeña como Decano de la Facultad Introductoria de Ciencias Informáticas (FICI) de la UCI. 